\section{Características clínico epidemiológicas y quirúrgicas de los pacientes con diagnóstico de carcinoma de mama}

\author{
Clinical epidemiological and surgical characteristics of patients \\ diagnosed with breast carcinoma
}

\section{Características epidemiológicas e cirúrgicas clínicas de pacientes diagnosticadas com carcinoma de mama}

\author{
Gilberto Areyan \\ gilbertoareyan2@hotmail.com \\ Recibido mayo 2018 / Revisión junio 2018 / Aceptado 1 de agosto 2018
}

\begin{abstract}
RESUMEN
Introducción: El cáncer de mama es la patología tumoral maligna de mayor incidencia en todo el mundo y está en crecimiento en países en vías de desarrollo. Objetivo: Analizar las características clínicas, epidemiológicas y quirúrgicas de los pacientes con diagnóstico de carcinoma de mama en el servicio "Dr. José Antonio Gubaira" de la Ciudad Hospitalaria Dr. Enrique Tejera durante el periodo enero 2017- junio 2018. Metodología: Se trata de un estudio descriptivo, con un diseño no experimental, transversal y prospectivo. La muestra fue de tipo no probabilística deliberada constituida por 60 pacientes, como instrumento se utilizó la ficha de registro. Los resultados se presentaron en tablas de distribuciones de frecuencias. Resultados y Discusión: El tipo de carcinoma predominante fue el Adenocarcinoma Ductal Infiltrante (78,33\%). Los estadios IIB y el IIIB fueron los más frecuentes con un $26,67 \%$ por igual. Un $58,33 \%$ de los pacientes con diagnóstico de carcinoma recibió terapia de neoadyuvancia previa. El tipo de intervención predominante fue la Mastectomía Radical Modificada Tipo Madden. Conclusión: Tanto los hombres como mujeres pueden padecer esta patología y un gran porcentaje tuvo una evolución satisfactoria.
\end{abstract}

Palabras Clave: Carcinoma de mama; epidemiología; clínica y cirugía

\begin{abstract}
Introduction: Breast cancer is the malignant tumor pathology with the highest incidence worldwide and is growing in developing countries. It is characterized by the accelerated, disorderly and uncontrolled growth of cells with genetic alterations, which act in the cell cycle suppressing or stimulating their continuity. Objective: To analyze the clinical, epidemiological and surgical characteristics of patients diagnosed with breast carcinoma in the "Dr. José Antonio Gubaira" from the Dr. Enrique Tejera Hospital City during the period January 2017 - June 2018. Methodology: This is a descriptive observational study, with a non-experimental, transversal and prospective design. The sample was of a deliberate nonprobabilistic and voluntary type consisting of 60 patients treated in the service with diagnosis of breast carcinoma. The information collection technique was direct observation and as an instrument the registration form was used. The results were presented in frequency distribution tables. Results and Discussion: The predominant type of carcinoma was the infiltrating Ductal Adenocarcinoma (78.33\%). Stages IIB and IIIB were the most frequent with $26.67 \%$ equally. $58.33 \%$ of patients diagnosed with carcinoma received prior neoadjuvant therapy. The predominant type of intervention was the Modified Radical Mastectomy Madden Type. Conclusion: Both men and women can suffer from this pathology and a large percentage had a satisfactory evolution
\end{abstract}

Key words: breast carcinoma; epidemiology; clinic and surgery http://revistavive.org

ISSN: $2664-3243$

pp. $154-166$
GA: Servicio de Cirugía General "Dr. José Antonio Gubaira", Venezuela. 


\section{RESUMO}

Introdução: O câncer de mama é a patologia maligna mais comum em todo o mundo e está crescendo nos países em desenvolvimento. Objetivo: analisar as características clínicas, epidemiológicas e cirúrgicas das pacientes diagnosticadas com carcinoma de mama no "Dr. José Antonio Gubaira ", da cidade do Hospital Dr. Enrique Tejera, no período de janeiro de 2017 a junho de 2018. Metodologia: Trata-se de um estudo descritivo, com desenho não experimental, transversal e prospectivo. A amostra foi do tipo não probabilístico deliberado, composto por 60 pacientes, como instrumento foi utilizado o formulário de registro. Os resultados foram apresentados em tabelas de distribuição de frequências. Resultados e Discussão: $O$ tipo predominante de carcinoma foi o adenocarcinoma infiltrante ductal $(78,33 \%)$. Os estágios IIB e IIIB foram os mais frequentes, com $26,67 \%$ igualmente. $58,33 \%$ dos pacientes diagnosticados com carcinoma receberam terapia neoadjuvante prévia. O tipo de intervenção predominante foi a Mastectomia Radical Modificada Madden Type. Conclusão: Homens e mulheres podem sofrer dessa patologia e uma grande porcentagem teve uma evolução satisfatória.

Palavras-chave: carcinoma de mama; epidemiologia; clínica e cirurgia

\section{INTRODUCCIÓN}

$\mathrm{E}$ l cáncer de mama es una neoplasia caracterizada por crecimiento acelerado, desordenado y no controlado de las células con alteraciones genéticas, las cuales actúan en el ciclo celular suprimiendo o estimulando su continuidad. Es la patología tumoral maligna de mayor incidencia en todo el mundo y está en crecimiento en países en vías de desarrollo.

Esta patología es el cáncer más frecuente en las mujeres tanto en los países desarrollados como en los países en vías de desarrollo. De acuerdo a la Organización Mundial de la Salud (OMS), en los últimos 25 años se duplicó el número de nuevos casos anuales, aunque la mortalidad ha disminuido en países desarrollados debido a la realización de diagnósticos tempranos y tratamientos más efectivos $(1,2)$. Corresponde a casi un $25 \%$ de los cánceres diagnosticados en mujeres para 2012, es decir, millones de nuevos casos por año.

De los casi 3.5 millones de mujeres que mueren por cáncer anualmente 522.000 mueren por cáncer de mama, es la primera causa de mortalidad a nivel mundial en mujeres y la causa más frecuente de mortalidad por cáncer en mujeres en los países de ingresos bajos y medios, con 324.000 muertes, lo que representa el 14,3\% del total de casos (2).

En países desarrollados como Estados Unidos, Inglaterra y España su incidencia se incrementó de 1973 a 2004 aproximadamente $0,94 \%$ a $4 \%$ por año sobre todo en mujeres mayores de 55 años, con diagnósticos cada vez más tempranos y más localizados lo que ha permitido la disminución de la mortalidad y el aumento de la sobrevida (3).

En contraposición, en países subdesarrollados como los de América Latina este cáncer es más frecuente en mujeres de 40 a 75 años de edad. El enorme problema de salud que significa en la actualidad el cáncer de la mama se refleja en el hecho de que "en América Latina y el Caribe casi 300.000 mujeres mueren anualmente por esta enfermedad". En otras palabras, esta neoplasia causa la muerte de 83 mujeres por día, o sea, que cada hora fallecen tres mujeres víctimas del cáncer de la mama $(4,5)$. 
En Venezuela, el cáncer de mama es la principal causa de incidencia por cáncer en mujeres, corresponde al 16,38\% del total de nuevos casos de cáncer en mujeres para 2010. En el período de 1990-2010 se produjeron 58.093 casos, solo 558 casos en hombres (1\%). Dicha patología aumentó de 1.560 casos en 1990 a 4.073 casos en 2010, con un incremento anual de casi $5 \%$. La tasa de incidencia de cáncer de mama aumentó de $23,53 \%$ por cada 100.000 mujeres a $29,68 \%$ por cada 100.000 mujeres (3).

La incidencia para mujeres entre 20 a 34 años ha mostrado estabilidad en el tiempo; a partir de los 35 a los 60 años se evidencia un crecimiento en las curvas estadísticas, el ascenso es más pronunciado en el grupo etario de 50 a 55 años. A partir de esta edad, se aprecia un comportamiento bifásico caracterizado por un descenso de la incidencia entre los 60 a 69 años, luego un ascenso entre 70 y 74 años para terminar, descendiendo en los casos de mayores de 75 años. En Venezuela, el $41,37 \%$ del total de nuevos casos de cáncer de mama ocurre en mujeres en edad reproductiva (15 a 49 años). El grupo más significativamente afectado es el de 40 a 59 años $(57,45 \%)$. Las mujeres menores de 40 años corresponden al 13,11\% y para las mayores de 60 años es 29,44 \% (3).

Por lo anteriormente expuesto, se establece como objetivo general de la presente investigación: Analizar las características clínicas, epidemiológicas y quirúrgicas de los pacientes con diagnóstico de carcinoma de mama en el servicio "Dr. José Antonio Gubaira" de la Ciudad Hospitalaria Dr. Enrique Tejera, a fin de determinar cuáles son las características propias de las pacientes afectadas por dicha patología y develar los aspectos propios del manejo quirúrgico al que fueron sometidas.

Para lograr el objetivo planteado se establecieron como objetivos específicos: Caracterizar a las pacientes con diagnóstico de carcinoma de mama según la edad, estado civil, paridad, número de hijos; identificar el tipo de carcinoma, el estadio y el tipo de intervención más frecuente entre las pacientes incluidas en el estudio. Describir los hallazgos intraoperatorios de las pacientes e identificar la evolución a partir de la presencia de complicaciones y estancia hospitalaria.

Actualmente se observan pacientes más jóvenes que padecen esta patología, motivo por el cual se hace imperiosa la necesidad de diagnosticarlos y atenderlos en un estadio temprano, con la finalidad de aumentar su sobrevida y puedan llevar el resto de sus vidas de forma normal. Este trabajo se realiza el objetivo de identificar y sistematizar los diferentes grupos etarios, tipos histológicos, estadificación, comorbilidades y el recibimiento o no de tratamiento neoadyuvante, a fin de estudiar el pronóstico y la evolución de las pacientes con diagnóstico de cáncer de mama que ingresan en el servicio "Dr. José Antonio Gubaira" de la Ciudad Hospitalaria Dr. Enrique Tejera.

Es conocido por toda la sociedad médica internacional, que ante una problemática de mortalidad se deben realizar estudios clínicos, reportes estadísticos, tratamientos realizados, entre otros datos, que servirán de base para la toma de decisiones en la realización de nuevos programas, capacitaciones, estudios, campañas preventivas, necesarias para disminuirla. Por lo tanto, esta investigación será base fundamental para estos programas, puesto que, describirá detalladamente una base de datos de casos de cáncer de mama con toda la información actual y real según las historias clínicas de pacientes afectados por esta enfermedad. Además, beneficiará en primer lugar las personas que padecen 
esta patología, puesto que, la población conocerá de esta enfermedad y podrá sensibilizarse e incurrir en la prevención mermando las estadísticas de cáncer.

Entre los estudios que desarrollan la temática central de la presente investigación, se encuentra el trabajo realizado en España por Ocón y Cols en el año 2010 en los hospitales Universitario San Cecilio y Virgen de las Nieves de Granada, y Torrecárdenas de Almería, donde se estudiaron a 202 mujeres diagnosticadas e intervenidas por cáncer de mama que cumplían los criterios de inclusión establecidos (estado civil, nivel educativo, ocupación y clase económicosocial, así como número de embarazos, paridad, meses acumulados de lactancia, antecedentes familiares de cáncer de mama y consumo de alcohol y tabaco); fueron apareadas por edad y hospital con otras 260 mujeres intervenidas de patología no oncológica, ni ginecológica ni endocrina. La edad media al diagnóstico fue de $54,27 \pm 10,4$ años, la supervivencia global a los 5 años fue del $83,9 \%$ a los 10 años del $71 \%$, la mortalidad de la serie fue del 33,17\% (6).

En este mismo orden de ideas, una investigación realizada en España en el año 2015 , se estudió la calidad de vida en mujeres supervivientes entre 5 y 8 años al cáncer de mama, identificando las secuelas físicas y psicológicas que sufren; para ello se tomó como muestra a 120 pacientes cuya edad media fue de 51 a 76 años, $72,5 \%$ estaban casadas, $46,7 \%$ tenían 2 hijos, $38,3 \%$ habían sido sometidas a cirugía conservadora y el 58,3\% recibieron quimioterapia + radioterapia + terapia hormonal. En conclusión, tres de cada cuatro mujeres supervivientes entre 5 y 8 años al cáncer de mama, tienen alterada su calidad de vida que se ve afectada por las siguientes secuelas: problemas del brazo, estado físico, problemas de alimentación, problemas de la mama, insatisfacción de la imagen corporal, el estado emocional, el funcionamiento social y sexual (7).

En el año 2013, en el Hospital Nacional Almanzor Aguinaga Asenjo en Perú, buscaron establecer el perfil epidemiológico, clínico y anatomopatológico de pacientes con cáncer de mama, se estudiaron a 117 pacientes, cuyo grupo de edad más afectado fue entre los 41 y 50 años $(40,2 \%)$; resalta la presencia de patología mamaria benigna previa $(25,6 \%)$, presencia de menarquía temprana $(19,7 \%)$, el uso de anticoncepción oral $(26,5 \%)$ y el antecedente familiar de cáncer de mama $(29,1 \%)$. La presencia de masa fue el signo más frecuente $94,9 \%$. El tumor predominante en un $86,5 \%$ fue el carcinoma ductal infiltrante variedad NOS. En el estudio de inmunohistoquímica, el $52,1 \%$ de las muestras dio un resultado positivo para Receptor de estrógeno, un $39,3 \%$ para progestágeno y un $29,1 \%$ para la prueba de HERCEPT (8).

En el Hospital José Carrasco Arteaga en la ciudad de Cuenca, en el año 2014, se realizó un estudio sobre las características epidemiológicas y clínicas del cáncer de mama en un hospital tipo III. Se registraron 202 casos diagnosticados histológicamente, $99,51 \%$ de sexo femenino y $0,49 \%$ de sexo masculino. El rango de edad fue de 41 a 60 años, la prevalencia de obesidad y sobrepeso fue del $56,9 \%$, el $7,9 \%$ tuvo antecedentes personales de cáncer de mama y el 17,8\% antecedentes familiares. El 9,9\% tuvo menarquia temprana, nuliparidad en $20,4 \%$, edad tardía del primer parto $7,9 \%$, menopausia tardía 4,4\%. El 24\% recibió tratamiento hormonal. El $77,2 \%$ fueron diagnosticados por mamografía. Dentro del diagnóstico histológico, el carcinoma ductal fue el más frecuente con un 93,1\%. Como tratamiento se realizó la cirugía radical en un $53,4 \%$ y conservadora en $46,5 \%$, quimioterapia en $86,6 \%$, terapia 
biológica en 15,8\%, hormonoterapia en $59,4 \%$ y radioterapia en $46 \%$ (9).

En Venezuela, en el Hospital Universitario de Los Andes, durante el año 2014, se estudiaron los factores pronósticos y predictivos en el cáncer de mama con sobre-expresión del HER2/NEU. Para ello se analizaron a 605 pacientes de las cuales, de mediana edad 53,5 años. El diagnóstico de cáncer de mama fue más frecuente en los grupos etarios 46-50 años y 51-55 años (15,37\% y $15,86 \%$ respectivamente). Se le realizó inmunohistoquímica al $62,2 \%$ de los casos, $21,1 \%$ reportó sobre-expresión del HER2/neu, 56 casos corresponden a HER2/neu + 3, con predominio en las mujeres de 46 a 50 años, $62,5 \%$ con estado posmenopáusico. El estado ganglionar más frecuente fue el "N0" 39,2\% y "N1" 30,3\%. Tamaño tumoral "T2" 35,7\%, "T3" y “T4"19,6\% respectivamente. El 62,5\% reportan receptores hormonales negativos. El tipo histológico más frecuente carcinoma ductal infiltrante 93\%. El 60,7\% tuvo grado histológico G2, $50 \%$ recibieron neoadyuvancia + cirugía + adyuvancia. Se observó $92 \%$ de supervivencia al año del tratamiento y 83,2 $\%$ a los dos años (10).

En Barquisimeto, estado Lara, Uribe y Cols (11) en el año 2016, estudiaron a 33 pacientes con cáncer de mama, luminales $\mathrm{A}$ y B estadios I - II, usando el pequeño espécimen del tumor, se realiza estudio histológico, inmunohistoquímica y Oncotype. En el $94 \%$ fue una muestra suficiente para realizar Oncotype. 61\% fueron bajo riesgo, $26 \%$ riesgo intermedio, $16 \%$ alto riesgo. Si se hubiese escogido tratamiento de acuerdo al carácter clínicopatológico del tumor, 61\% hubiese recibido quimioterapia, $39 \%$ hormonoterapia, con resultado del Oncotype, solo $32 \%$ de pacientes recibieron quimioterapia y 55\% hormonoterapia, $1 \%$ con tumores $\mathrm{T} 1 \mathrm{a}-\mathrm{b}$,
Luminales A y escore de recurrencia bajo fueron operadas y colocadas en hormonoterapia adyuvante.

Se conocen bien, varios factores de riesgo del cáncer de mama. Sin embargo, en la mayoría de las mujeres afectadas, no es posible identificar factores de riesgo específicos. Los factores reproductivos relacionados a una exposición prolongada a estrógenos endógenos, como una menarquia precoz, una menopausia tardía y una edad madura cuando el primer parto figura entre los factores de riesgo más importantes del cáncer de mama. Las hormonas exógenas también conllevan un mayor riesgo, por lo que las usuarias de anticonceptivos orales y de terapia de sustitución hormonal tienen más riesgo que las mujeres que no usan esos productos. La lactancia materna tiene una derivación de protección contra este padecimiento (12). Se ha calculado la contribución de diversos factores de riesgo modificables, exceptuando los factores reproductivos, a la carga mundial de cáncer de mama. Los autores consuman que el $21 \%$ de todas las muertes por cáncer de mama registradas en el mundo, son atribuibles al consumo de alcohol, el sobrepeso, la obesidad y sedentarismo.

Esa proporción fue mayor en los países de ingresos altos (27\%) y el factor más importante, fueron el sobrepeso y la obesidad. En los países de ingresos bajos y medios, la proporción de cánceres de mama atribuibles a esos factores de riesgo fue del $18 \%$ y la falta de actividad física fue el factor determinante más importante $(10 \%)(12,13)$.

La diferente incidencia del cáncer de mama en los países desarrollados y los países en desarrollo, puede explicarse en parte por los efectos de la alimentación, unidos a la mayor edad del primer embarazo, el menor número de partos y el acortamiento de la lactancia. El progresivo acogimiento de modos de vidas 
occidentales en los países de ingresos bajos y medios, es un determinante importante del aumento de la incidencia de cáncer de mama en esos países.

Con respecto a los factores pronósticos, se dividen en aquellos que son dependientes del tumor y dependientes del paciente. Los dependientes del tumor son: afectación ganglionar, tamaño tumoral, tipo histológico, diferenciación histológica, expresión de receptores hormonales, localización del tumor, angiogénesis, ploidía, índice de proliferación, índice de apoptosis, expresión de receptores de crecimiento epidérmico (EGFR), enzimas proteolíticas de la matriz extracelular, moléculas de adhesión, reguladores del ciclo celular. Entre los factores pronósticos dependientes del paciente están: sexo, edad, índice de masa corporal, situación hormonal, situación inmunológica, factores psicológicos (12).

La estadificación del cáncer de mama provee información respecto al pronóstico y orienta el método terapéutico a utilizar. Los estudios de imagen en la actualidad son un complemento para evaluar el tamaño del tumor, la presencia de los ganglios y las metástasis (13). El sistema TNM es una estrategia para agrupar a las pacientes con respecto a su pronóstico. Además, se pueden establecer decisiones terapéuticas, en parte de acuerdo a su categoría, respecto a su clasificación linfonodal, condición menopáusica y estado general de la paciente.

La patología mamaria maligna se puede dividir en: carcinomas no invasores e invasores de la mama. Entre los carcinomas no invasores de la mama está el carcinoma lobulillar in situ y el carcinoma ductal in situ.

El carcinoma lobulillar in situ (CLIS), está constituido por una proliferación de células epiteliales atípicas, pequeñas y uniformes que rellenan y distienden los lobulillos, conservando la arquitectura lobulillar. Su hallazgo histológico se encuentra en tejidos glandulares mamarios que mascroscopicamente son normales o con displasia, es decir, no tienen manifestación macroscópica. El carcinoma ductal in situ (CDIS) es una proliferación de células más grandes que las que forman el CLIS, con núcleos hipercromáticos, en algunos tipos más uniformes como en grados bajos o más pleomórficos como en grados altos. A su vez, en el CDIS hay varios subtipos los cuales son: solido, cribiforme, papilar y comedociano (14).

Entre los carcinomas invasores se encuentran: El carcinoma ductal infiltrante, el cual es el tumor maligno más frecuente, representando del 65 al 80\% de todos los carcinomas de mama. Comprende todos aquellos tumores malignos de origen ductal que no adoptan en forma absoluta, un patrón histológico de crecimiento especifico. El carcinoma lobulillar infiltrante representa de 10 a $14 \%$ de todos los carcinomas infiltrantes de mama. Se ha visto asociado a carcinoma lobulillar in situ en $60 \%$ de los casos. Su presentación clínica y mamográfica no es precisa, pudiendo presentarse como sutil endurecimiento más o menos localizado. Existen otros tipos de carcinomas los cuales son menos frecuentes, entre ellos se encuentran: carcinoma medular, tubular, mucinoso, papilar y cribiforme (14).

Con respecto al tratamiento quirúrgico, la conducta ha cambiado, ya que durante muchos años el tratamiento de elección era ser lo más radical posible, sin embargo, actualmente el tratamiento está orientado hacia la conservación de la mama y el tejido linfático ganglionar axilar. La cirugía conservadora es la extirpación parcial de la mama con o sin piel, la exéresis del complejo areola pezón también se considera tratamiento conservador, se realiza con la finalidad de 
obtener una mínima mutilación con buen resultado estético, obtener una máxima información oncológica y control de la enfermedad local. La cirugía radical (mastectomía) es la extirpación quirúrgica de la glándula mamaria: piel, complejo areola-pezón y todo el tejido glandular mamario (12).

Entre las cirugías conservadoras de la mama se encuentra la tumorectomía, que es la extirpación de la tumoración con márgenes de seguridad. La segmentectomía que es la exéresis de un segmento de la mama, que incluya la lesión. Y la cuadrantectomía es la extirpación de un cuadrante de la mama, es más amplia que la segmentectomía ya que incluye la piel (12).

Entre los tipos de cirugía radical se encuentra la mastectomía simple y consiste en la extirpación de la mama y la fascia pectoral, indicada en tumores phyllodes malignos, también es utilizada de forma paliativa en casos de edad avanzada. La mastectomía radical también llamada de tipo Halsted, se realiza exéresis de la mama, la fascia pectoral mayor, músculos pectoral mayor y menor, gran cantidad de piel y todos los niveles ganglionares axilares (I, II y III). La mastectomía radical modificada, también llamada de tipo Madden, se realiza extirpación del tejido mamario, la fascia pectoral mayor, y exéresis de los niveles ganglionares I y II. Actualmente, es la técnica radical más utilizada. Existen otros tipos de mastectomías menos frecuentes los cuales son: la radical modificada tipo Merola-Patey, ahorradora de piel, subcutánea, radical ampliada, supraradical $(12,13)$.

El objetivo de esta investigación es analizar las características clínicas, epidemiológicas y quirúrgicas de los pacientes con diagnóstico de carcinoma de mama en el servicio "Dr. José Antonio Gubaira" de la Ciudad Hospitalaria Dr. Enrique Tejera.

\section{MATERIALES Y MÉTODOS}

I nvestigación descriptiva con un diseño transversal y prospectivo. La población representada por 148 pacientes atendidas en el servicio de Cirugía A. La muestra, de tipo no probabilística deliberada está constituida por 60 pacientes con diagnóstico histológico de carcinoma de mama, siendo este el único criterio de inclusión, independientemente de su etiología, presentación, gravedad y pronóstico.

Entre los criterios de exclusión, se establecieron aquellos pacientes que no presenten con diagnóstico confirmado por biopsia de tejido mamario, aquéllos que se hayan negado a formar parte de dicha investigación, pacientes cuyo origen de cáncer primario no sea mama y los que ingresen que diagnóstico de patología benigna mamaria.

Se empleó como técnica de recolección de información la observación directa y como instrumento se utilizó la ficha de registro. Para el análisis de los datos, se sistematizaron en una tabla maestra realizada mediante Microsoft@ Excel, presentando los resultados a partir de las técnicas estadísticas descriptivas univariadas en tablas de distribuciones de frecuencias. Se utilizó el programa estadístico Statgraphics Plus 5.1.

Se solicitó el consentimiento informado de las pacientes y la investigación fue autorizada por el Comité de Ética de la Institución.

\section{RESULTADOS Y DISCUSIÓN}

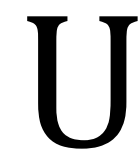

na vez analizadas las características clínicas, epidemiológicas y quirúrgicas de los 60 pacientes con diagnóstico de carcinoma de mama, los resultados obtenidos reflejan lo siguiente: 
Tabla 1.- Pacientes con diagnóstico de carcinoma de mama según la edad, estado civil, paridad, número de hijos.

\begin{tabular}{lll}
\hline Edad (años) & f & \% \\
\hline $28-44$ & 18 & 30 \\
$45-60$ & 26 & 43,33 \\
$>60$ & 16 & 26,67 \\
Sexo & $\mathbf{F}$ & $\mathbf{\%}$ \\
Femenino & 56 & 93,33 \\
Masculino & 4 & 6,67 \\
Estado civil & $\mathbf{F}$ & $\mathbf{\%}$ \\
Soltera (o) & 42 & 70 \\
Casada (o) & 14 & 23,33 \\
Divorciada (o) & 4 & 6,67 \\
Paridad & $\mathbf{F}$ & $\mathbf{\%}$ \\
Multípara & 56 & 93,33 \\
No aplica & 4 & 6,67 \\
Número de hijos & $\mathbf{F}$ & $\mathbf{\%}$ \\
0 & 4 & 6,66 \\
1 & 6 & 10,53 \\
2 & 25 & 43,86 \\
$3-4$ & 23 & 38,33 \\
5 & 2 & 3,51 \\
Total & $\mathbf{6 0}$ & $\mathbf{1 0 0}$ \\
\hline
\end{tabular}

Con respecto a la edad, el grupo mayor fue de 45 a 60 años con un $43,33 \%$ seguido de aquellos pacientes con 28 y 44 años 30\%; el sexo femenino predominó; estado civil un $70 \%$ de la muestra eran solteros; todas las mujeres eran multíparas. El tipo de carcinoma predominante fue el Adenocarcinoma
Ductal Infiltrante con un 78,33\% (47 casos), seguido del Adenocarcinoma Ductolobulillar 13,33\% (8 casos). En cuanto al estadio se tiene que el IIB y el IIIB fueron los más frecuentes con un $26,67 \%$ por igual (16 casos cada estadio), seguido del estadio IIA $(21,67 \% \quad(13$ casos). 
Tabla 2. Identificación del tipo de carcinoma, el estadio y el tipo de intervención más frecuente entre las pacientes incluidas en el estudio.

\begin{tabular}{lll}
\hline ADC Ductal infiltrante & 47 & 78,33 \\
\hline ADC Ductolobulillar & 8 & 13,33 \\
ADC Ductal in situ & 3 & 5 \\
Tipo de carcinoma & $\mathbf{F}$ & $\mathbf{\%}$ \\
Carcinoma medular & 2 & 3,33 \\
Estadio & $\mathbf{F}$ & $\mathbf{\%}$ \\
I & 1 & 1,67 \\
IIA & 13 & 21,67 \\
IIB & 16 & 26,67 \\
IIIA & 11 & 18,33 \\
IIIB & 16 & 26,67 \\
TIS & 3 & 5 \\
Inmunohistoquímica & $\mathbf{F}$ & $\mathbf{\%}$ \\
(+) Receptores estrógeno y progesterona & 10 & 16,67 \\
(+) Receptores Ki-67 & 3 & 5 \\
(+) Receptores Her 2 & 2 & 3,33 \\
(+) Receptores progesterona y (+) Ki-67 & 1 & 1,67 \\
No Realizada & 44 & 73,33 \\
Tipo de intervención & $\mathbf{F}$ & $\mathbf{\%}$ \\
Mastectomía radical modificada tipo Madden & 52 & 86,67 \\
Mastectomía simple & 3 & 5 \\
Mastectomía subcutánea & 1 & 1,67 \\
No se operó & $\mathbf{6 0}$ & 6,67 \\
Total & $\mathbf{1 0 0}$ \\
\hline
\end{tabular}


Tabla 4. Hallazgos intra-operatorios de los pacientes intervenidos quirúrgicamente, Ciudad Hospitalaria "Dr. Enrique Tejera".

\begin{tabular}{lll}
\hline Cuadrantes superior interno y externo & $\mathbf{1 6}$ & 26,67 \\
\hline Cuadrante superior externo & 13 & 21,67 \\
Lesión periareolar & 12 & 20 \\
4 Cuadrantes & 5 & 8,33 \\
Cuadrante superior interno & 4 & 6,67 \\
NR & 4 & 6,67 \\
Cuadrante Inferior interno & 2 & 3,33 \\
Retroareolar & 2 & 3,33 \\
Cuadrante inferior externo & 1 & 1,67 \\
Infiltra pectoral mayor, menor y parrilla costal & 1 & 1,67 \\
Infiltración ganglionar & $\mathbf{F}$ & $\mathbf{9}$ \\
Si & 47 & 78,33 \\
No & 13 & 21,67 \\
Total & $\mathbf{6 0}$ & $\mathbf{1 0 0}$ \\
\hline
\end{tabular}

Fuente: Datos de la investigación

Tabla 5. Identificación de la evolución de los pacientes con carcinoma de mama intervenidos, Ciudad Hospitalaria "Dr. Enrique Tejera".

\begin{tabular}{lll}
\hline Presentes & 15 & 25 \\
\hline Ausentes & 45 & 75 \\
Total & $\mathbf{6 0}$ & $\mathbf{1 0 0}$ \\
Tipo de complicación (n=15) & $\mathbf{F}$ & $\mathbf{\%}$ \\
ISQ & 5 & 8,33 \\
Recidiva en piel & 5 & 8,33 \\
Miasis & 2 & 3,33 \\
Recidiva en axila & 2 & 3,34 \\
Hematoma & 1 & 1,67 \\
Linfadenitis & 1 & 1,67 \\
Embarazo 29 sem & 1 & 1,67 \\
Mastectomía simple previa & 1 & 1,67 \\
Reintervención & 1 & 1,67 \\
Evolución & $\mathbf{F}$ & $\mathbf{\%}$ \\
Buena & 50 & 83,33 \\
Regular & 4 & 6,67 \\
Mala & 3 & 5 \\
No reportado & 3 & 5 \\
Estancia & $\mathbf{F}$ & $\mathbf{\%}$ \\
$\leq 7$ & 53 & 88,33 \\
$8-15$ & 5 & 8,33 \\
$>15$ & 2 & 3,33 \\
Total & 60 & 100 \\
\hline
\end{tabular}

De los 60 pacientes con carcinoma de mama, se registró una edad promedio de 51,9 años $\pm 1,70$, con una mediana de 53 años, una edad mínima de 28 años, una edad máxima de 77 años y un coeficiente de variación de $25 \%$ (serie homogénea entre sus datos), siendo más frecuente el grupo de 45 a 60 años con un 43,33\% (26 
casos), seguido de aquellos pacientes con 28 y 44 años, 30\% (18 casos). Dicha estadística se compagina con los datos presentados en investigaciones internacionales y nacionales. Concuerda con la investigación realizada en España por Ocón y Cols en el año 2010, cuya media al diagnóstico fue de 54,27 $\pm 10,4$ años (6) y el estudio realizado por Ruiz (7) en el año 2015, también en España, donde la edad media fue de 51,76 años.

Según informes, el grupo más afectado en patología mamaria maligna es el de 40 a 59 años, $57.45 \%$. En cuanto al sexo el femenino predominó un 93,33\% (56 casos) y se encontraron 4 casos masculinos, 6,67\%.

La edad promedio del sexo femenino fue de 52,02 años +/- 3,37, levemente mayor a la edad promedio del sexo masculino que fue de 50,25 años +/- 3,65, sin embargo, no se encontró una diferencia estadísticamente significativa entre ambos promedios $(\mathrm{t}=0,26 ; \mathrm{P}=0,7983>0,05)$. Esto difiere de la estadística presentada por Torres (9), en un estudio realizado en el Hospital José Carrasco Arteaga en la ciudad de Cuenca en el año 2014, donde en 202 casos diagnosticados, el sexo femenino predominó en un $99,51 \%$ y el masculino en un 0,49\%, difiriendo así de los resultados obtenidos en esta investigación que el sexo masculino tuvo un $6,67 \%$.

En relación al estado civil, un $70 \%$ de la muestra eran solteros (42 casos); todas las mujeres eran multíparas (56 casos) y fueron más frecuentes aquellos pacientes con 2 hijos 43,86\% ( 25 casos), seguidos de aquellos que tenían entre 3 y 4 hijos (23 casos). En un estudio realizado en España (7) se evidencian diferencias con los resultados en la presente investigación ya que predominaban las pacientes casadas en un $72,5 \%$, no obstante, coincide en que la mayoría de las pacientes tenían dos hijos, $46,7 \%$.
El tipo de carcinoma predominante fue el Adenocarcinoma Ductal Infiltrante con un 78,33\% (47 casos), seguido del Adenocarcinoma Ductolobulillar 13,33\% (8 casos), coincidiendo con las estadísticas presentadas en Perú (8), donde el tumor predominante en un $86,5 \%$ fue el carcinoma ductal infiltrante en el Hospital José Carrasco Arteaga, la investigación de Torres (9) arrojó un 93,01\% para el ductal infiltrante y en Venezuela, Rodríguez (10) encontró que el tipo histológico más frecuente fue el ductal infiltrante representado un $93 \%$.

En referencia al estadio, se tiene que el IIB y el IIIB fueron los más frecuentes con un $26,67 \%$ por igual (16 casos cada estadio), seguido del estadio IIA 21,67\% (13 casos). Difiere un poco de lo presentado por Rodríguez (10) donde la mayoría de los pacientes llegaban en estadios tempranos, siendo el estadio ganglionar más frecuente fue el "N0" $39,2 \%$ y "N1" 30,3 \%. Tamaño tumoral “T2" 35,7 \%, “Т3" у “Т4"19,6 \% respectivamente.

En la actual investigación, la mayoría de los pacientes ingresaron en estadios muy avanzados de la enfermedad y esto puede obedecer a la misma situación país: déficit de centros oncológicos, déficit de quimioterapia y radioterapia perjudicial al paciente, a su evolución satisfactoria $\mathrm{y}$ pronta mejoría.

En lo correspondiente a la inmunohistoquímica de los 16 pacientes que la refirieron, se tiene que el resultado más frecuente fue la expresión de receptores de estrógeno y progesterona (10 casos). Difiriendo también de otra investigación donde se le realizó inmunohistoquímica al 62,2 \% de los casos, 21,1\% reportó sobre-expresión del HER2/neu, 56 casos corresponden a HER2/neu +3; con predominio en las mujeres de 46 a 50 años, $62,5 \%$ con estado posmenopáusico (10). 
El tipo de intervención predominante fue la Mastectomía Radical Modificada Tipo Madden+ Vaciamiento Ganglionar Axilar 86,67\% (52 casos); seguida de la Mastectomía Simple 5\% (3 casos). Resultados que coinciden plenamente con los datos ofrecidos por Torres (9), donde el tratamiento quirúrgico que predominó en ese centro hospitalario fue la cirugía radical por encima de la conservadora con un $53,4 \%$. Un 58,33\% de los pacientes con diagnóstico de carcinoma recibió terapia de neoadyuvancia previa (35 casos), de los cuales predominaron aquellos que recibieron 8 sesiones 57,14\% (20 casos). Datos que son similares a la investigación realizada por Rodríguez (10), en donde el $50 \%$ de los pacientes recibieron terapia de neoadyuvancia. Con respecto al número de sesiones de neoadyuvancia (8 sesiones), este número tan elevado de sesiones puede obedecer al mismo colapso de las instituciones públicas para resolver este tipo de pacientes y obliga en cierta forma al oncólogo médico, a dar más sesiones de neoadyuvancia de las necesarias mientras el paciente espera resolución quirúrgica oportuna.

En cuanto a la localización del carcinoma, se tiene que fueron más frecuentes aquellos localizados en los Cuadrantes Superior Interno y Externo con un $26,67 \%$ (16 casos), seguidos de aquellos pacientes en los que el carcinoma se encontraba localizado en el Cuadrante Superior Externo 21,67\% (13 casos), $78,33 \%$ de los pacientes presentó infiltración ganglionar (47 casos). Datos que son propios de la investigación y no están descritos en la literatura revisada. Sólo un $25 \%$ de los pacientes intervenidos presentó complicaciones (15 casos). Del total de los pacientes ingresados, un predominante $83,33 \%$ de los pacientes presentó una evolución favorable o buena.

\section{CONCLUSIÓN}

onsiderando las características
clínicas, epidemiológicas y
quirúrgicas de los pacientes con diagnóstico de carcinoma de mama en el servicio "Dr. José Antonio Gubaira” de la Ciudad Hospitalaria Dr. Enrique Tejera, se determina entonces que tanto los hombres como mujeres pueden padecer esta patología y un gran porcentaje tuvo una evolución satisfactoria.

- Conflicto de intereses: Ninguno declarado por el autor.

- Fuente de financiamiento: Autofinanciado.

- Agradecimientos: Ninguno declarado por el autor.

\section{REFERENCIAS}

1. Organización Mundial de la Salud. Cáncer de mama: prevención y control [Internet]. OMS 2017 [citado 201728 de febrero]. Disponible en:

http://www.who.int/topics/cancer/breastcancer/ es/

2. Torres L, Barragán J, Becerra G, González N, Mainero F, Mijares A, et al. Guía de práctica clínica. Diagnóstico y tratamiento del cáncer de mama en segundo y tercer nivel de atención [Internet]. México: Secretaria de Salud; 2009 [citado 201728 de febrero]. Disponible en: http://www.cenetec.salud.gob.mx/descargas/gp c/CatalogoMaestro/232_IMSS

3. Capote L, García I, Billalta D, Alvins A, De Abreu M, Manso A, et al. Guía para el cáncer de mama en Venezuela [Internet]. Caracas: Sociedad Anticancerosa de Venezuela; 2015 [citado 201728 de febrero]. Disponible en: http://sociedadanticancerosa.org.ve/wpcontent/uploads/2015/11/Gui\%CC\%81a-parael-Ca\%CC\%81ncer-de-MamaenVenezuela.pdf

4. Robles S, Galanis E. El cáncer de mama en América Latina y El Caribe. Informar sobre las opciones. Rev Pan de Sal Pub (Washington). 2002; 12 (2): 141-143

5. Falcones K. Caracterización del cáncer de mama en el Hospital Abel Gilbert Pontón. Periodo de diciembre 2013 a diciembre 2014 [Internet]. Guayaquil: Universidad de Guayaquil; 2014 [citado 201702 de marzo]. Disponible en: http://repositorio.ug.edu.ec/bitstream/redug/10 708/1/CANCER\%20DE\%20M 
6. Ocón O, Fernández M, Pérez S, Dávila C, Hernández J, Olea N. Supervivencia en cáncer de mama tras 10 años de seguimiento en las provincias de Granada y Almería. Rev Esp de Sal Pub (Madrid). 2010; 84 (6): 705-715. DOI: $10.1590 /$ S1135-57272010000600003

7. Ruiz P. Estudio sobre la calidad de vida en mujeres supervivientes al cáncer de mama [Internet]. Murcia: Universidad de Murcia; 2015 [citado 201702 de marzo]. Disponible en: https://digitum.um.es/xmlui/bitstream/10201/45 520/1/TESIS\%20COMPLET

8. Pinto-Larrea I. Perfil epidemiológico, clínico y anatomopatológico del cáncer de mama. Rev. Cuerpo Med. HNAAA 2013; 6 (1): 8-15

9. Torres I. Características epidemiológicas y clínicas del cáncer de mama. Hospital José Carrasco Arteaga, Cuenca 2014 [Internet]. Universidad de Cuenca; 2014 [citado 201702 de marzo]. Disponible en: http://dspace.ucuenca.edu.ec/bitstream/12345 6789/21274/1/TESIS63.pdf
10. Rodríguez N, La Cruz C, Márquez M, López F, Borges R. Factores pronósticos y predictivos en el cáncer de mama con sobre-expresión del her-2/. Rev Venez Oncol 2014; 26(3):175-186. Disponible en: https://www.redalyc.org/pdf/3756/3756339700 03.pdf

11. Uribe J. Impacto del resultado del Oncotype obtenido mediante aguja gruesa en el tratamiento neoadyuvante de pacientes con cáncer de mama. Rev Venez Oncol. 2016; 28(1):9-21

12. Sierra A, Piñero A, Illana J. Cirugía de la mama. Madrid:Aran Ediciones; 2006

13. Bland K, Copeland III E. La mama: Manejo multidisciplinario de las enfermedades benignas y malignas. 3ra ed. Buenos Aires: Médica Panamericana; 2007.

14. Hernández G, Bernardello E, Pinotti J, Barrios A. Cáncer de mama. 2da ed. Caracas: McGraw-Hill Interamericana; 2007 